\title{
Keine Dosisanpassung bei niereninsuffizienten Diabetikern
}

— „Etwa 40\% der Diabetiker haben eine Nierenschädigung, die den behandelnden Arzt vor die Frage stellt: Kann ich ein orales Antidiabetikum noch geben oder nicht", sagte Prof. Stephan Martin, Düsseldorf.

Metformin ist bei Niereninsuffizienz kontraindiziert. Die bisher verfügbaren Gliptine sind nur bis zu einer glomerulären Filtrationsrate von $60 \mathrm{ml} / \mathrm{min}$ zugelassen oder die Dosis muss reduziert werden. „Daher müssen wir niereninsuffizienten Diabetikern häufig schon in einer frühen Phase Insulin geben", sagte Martin.

Ein Ausweg könnte der neue DPP-4Hemmer Linagliptin (Tradjenta ${ }^{\circledR}$ ) sein. Anders als Saxa-, Vilda- und Sitagliptin wird die Substanz nur zu etwa 5\% über die Niere und sonst weitgehend unverändert über die Galle und den Darm ausgeschieden. Daher muss die Dosis bei Nierenfunktionseinschränkungen nicht angepasst werden. Auch bei Leberfunktionsstörungen, selbst bei schweren, ist keine Dosisanpas-

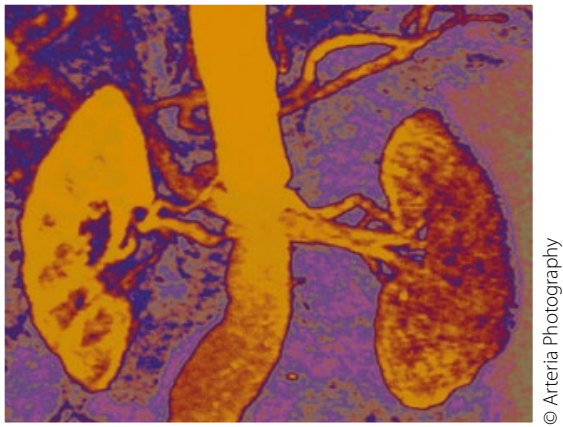

Ca. $40 \%$ der Diabetiker haben eine Nierenschädigung.

sung nötig (Friedrich $C$ et al. Diabetologie und Stoffwechsel 211; 6: S76, Poster 217).

Dass Linagliptin den bisher verfügbaren Gliptinen von der Wirkung her gleichgestellt ist, hat eine Phase-III-Studie ergeben (Del Prato et al. Diabetes, Obesity and Metabolism 2011; 13; 258-267). Wie Martin berichtete, wurden darin $z$. T. antidiabetikanaive Typ-2-Diabetiker, die einen Ausgangs- $\mathrm{HbA}_{1 \mathrm{c}}$ von etwa $8 \%$ hatten, entwe- der mit Placebo $(n=167)$ oder Linagliptin in Monotherapie behandelt $(n=336)$. Nach 24 Wochen war der $\mathrm{HbA}_{1 c}$-Wert (primärer Endpunkt) mit dem neuen Gliptin im Vergleich zur Placebogruppe im Mittel um $0,69 \%$ gefallen. Auch Nüchtern- und postprandialer Blutzucker sowie Betazellfunktionsrate sind mit dem Verumpräparat signifikant besser ausgefallen, so Martin. Unerwünschte Wirkungen und Hypoglykämien durch das Gliptin habe es nicht gegeben.

Linagliptin wird einmal täglich eingenommen (5 mg). Die Monotherapie ist zugelassen, wenn der Blutzucker durch Diät und Bewegung allein nur unzureichend kontrolliert werden kann und wenn Metformin wegen Unverträglichkeit nicht geeignet oder aufgrund von Nierenfunktionsstörungen kontraindiziert ist.

- Sarah Pampel

Quelle: Pressekonferenz auf der DDG-

Jahrestagung, Leipzig, Juni 2011 (Veran-

stalter: Boehringer Ingelheim und Lilly)

\section{Schmerztherapie}

\section{Austausch starker Opioide ist problematisch}

- Grundsätzlich gilt die automatische Austauschpflicht von Originalmedikamenten auf Generika auch für starke Opiode, sofern dies durch das Aut-idem-Kreuz auf dem BtMVV-Rezept nicht ausgeschlossen worden ist. Doch der Austausch ist höchst problematisch, betonte Prof. Dr. rer. nat. Harald Schweim, Bonn, selbst wenn alle Kriterien für den Austausch erfüllt sind. Eine unterschiedliche Retard-Galenik kann z. B. zu einer unterschiedlichen Freisetzung des Wirkstoffs in Verbindung mit anderen Substanzen wie z. B. Alkohol führen. Zudem werden in Bezug auf die Bioverfügbarkeit Abweichungen von bis zu 45\% zwischen zwei Präparaten akzeptiert.

Ein Austausch zweier als bioäquivalent geltender starker Opioide kann daher mit Veränderungen der Plasmaspiegel, mit einem schnelleren Wirkeintritt oder einer deutlich verkürzten Wirkdauer einherge- hen. Außerdem können Abweichungen von Farbe, Form und Geschmack der Tabletten die Patientencompliance beeinträchtigen. „Meiner Meinung nach sind starke Analgetika überhaupt nicht für den Austausch geeignet", sagte Schweim. Ein Austausch entspreche fast immer einer Neueinstellung.

\section{Substitution im Praxisalltag weit verbreitet}

Die Realität sieht allerdings anders aus. In einer Studie im Auftrag der Deutschen Gesellschaft für Schmerztherapie e.V. (DGS) berichteten $84 \%$ der befragten Ärzte über nicht medizinisch indizierte Substitutionen stark wirksamer Opioide in der Praxis. Bei fast zwei Dritteln der betroffenen Patienten verschlechterte sich der Krankheitsverlauf signifikant und die Therapie musste verändert werden, berichtete Schweim.
Starke Opioide sollten bei Patienten mit opioidpflichtigen Schmerzen nach individuellen Kriterien ausgewählt werden. Bei chronischen Schmerzen sollten von Beginn an retardierte Präparate eingesetzt werden, so Scheim, die möglichst über die gesamte Nacht wirken. Vorteilhaft sind Substanzen, die bei der Verstoffwechslung keine relevanten aktiven Metabolite bilden, die auch für ältere Patienten geeignet sind und deren Dosis individuell der Schmerzstärke angepasst werden kann. Durch Einstellung auf die Fixkombination Oxycodon/Naloxon (Targin ${ }^{\circledR}$ ) könnten Darmfunktionsstörungen, die häufigste und stärkste Nebenwirkung starker Opioide, umgangen werden.

\footnotetext{
- Roland Fath

Quelle: Apotheken-Update „Starke Opioide“, Frankfurt am Main, Juni 2011 (Veranstalter: Mundipharma)
} 